\title{
Level 3 Milestone Report: April 2020
}

\author{
Stephanie J Parker
}

April 2020

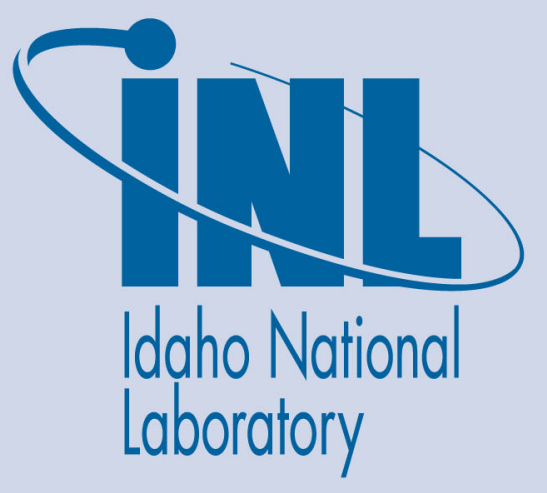

The INL is a U.S. Department of Energy National Laboratory operated by Battelle Energy Alliance 


\title{
Level 3 Milestone Report: April 2020
}

\author{
Stephanie J Parker
}

April 2020

Idaho National Laboratory Idaho Falls, Idaho 83415

http://www.inl.gov

Prepared for the

U.S. Department of Energy

Under DOE Idaho Operations Office

Contract DE-AC07-05ID14517 


\section{Level 3 Milestone Report: April 2020}

\section{HPC Utilization Data for NSUF/NE5 Projects from October 1, 2019, through March 31, 2020}

\section{M3UF-20IN0214013}

Idaho National Laboratory (INL)'s High Performance Computing (HPC) resources provide scientific computing capabilities to support INL and DOE-NE's efforts in advanced modeling and simulation. The HPC systems support a wide range of research activities, including performance of materials in harsh environments (including the effects of irradiation and high temperatures), modeling of geomechanical changes in subsurface flow, performance of advanced nuclear reactors, and multiscale analysis of nuclear fuel performance.

The Nuclear Science User Facilities (NSUF) program combines access to nuclear science R\&D capabilities and staff expertise with novel ideas provided by external contributors (universities, laboratories, and industry). These collaborations represent the cutting edge of nuclear technology research and stimulate cooperative research among user groups conducting basic and applied research. INL's HPC resources support universities and other external collaborators as part of the NSUF Program.

Current HPC resources include the following:

- Sawtooth: INL's newest supercomputer, an HPE SGI 8600 distributed memory system, deployed in March 2020 in the Collaborative Computing Center: 99,792 cores, 395 TB of total memory, and 0.56 PF of GPU capabilities. The current LINKPACK rating for Sawtooth is about 6 petaflops from both CPUs and GPUs. Ranked 37th on November 2019's list of the Top500 fastest supercomputers in the world, it has achieved the highest ranking of any INL supercomputer.

- Falcon: an SGI ICE X distributed-memory system: 34,992 cores, 121 TB of total memory, high-speed FDR interconnect network, and high-speed disk storage. The current LINPACK rating for Falcon is 1.087 petaflops.

- Lemhi: a Dell PowerEdge distributed-memory system: 20,160 cores, 94 TB of total memory, Omni-Path interconnect network, and high-speed storage. The LINPACK rating for Lemhi is 1.002 petaflops.

- Galena: a NVIDIA DGX-1 system for machine learning and artificial intelligence.

INL also leverages visualization resources such as a 3D immersive CAVE environment and various power-wall systems to help researchers understand their data and communicate complex science to stakeholders, as well as to the general public. 
The following table shows compute utilization in million core-hours for all machines from October 1, 2019, through March 31, 2020:

\begin{tabular}{|l|l|c|}
\hline \multicolumn{2}{|c|}{ Project } & Million \\
\hline ANSUF & ATR NSUF Project (Legacy Project) & Core-Hours \\
\hline ART & Reactor Engineering & 0.091 \\
\hline ATR & Advanced Reactor Technologies & 3.145 \\
\hline CASL & Consortium for Advanced Simulation of Light Water Reactors & 9.396 \\
\hline EEST & Energy \& Environment Science \& Technology & 16.787 \\
\hline EFRC & Energy Frontier Research Centers & 31.622 \\
\hline FCRD & Fuel Cycle Research \& Development & 3.981 \\
\hline HPC & High Performance Computing support & 6.352 \\
\hline ISO & Isotope project & 0.229 \\
\hline IUC & Idaho University Consortium & 0.074 \\
\hline LDRD & Laboratory Directed Research \& Development & 14.845 \\
\hline LWRS & Light Water Reactor Sustainability & 6.772 \\
\hline MMM & Materials Minimization \& Management & 2.994 \\
\hline MOOSE & MOOSE-based code development & 5.440 \\
\hline NEAMS & Nuclear Energy Advanced Modeling \& Simulation & 8.587 \\
\hline NEUP & Nuclear Energy University Program & 21.269 \\
\hline NHS & National \& Homeland Security & 8.881 \\
\hline NST & Nuclear Science \& Technology & 3.738 \\
\hline NSUF & Computer allocation through Nuclear Science User Facilities & 15.531 \\
\hline OTHER & & 4.835 \\
\hline STRUC & Structural Analysis / Facilities \& Site Services & 0.000 \\
\hline TREAT & Transient Reactor Test Facility & 0.129 \\
\hline VERA & VERA User Group members & 4.233 \\
\hline VTR & Versatile Test Reactor & 7.247 \\
\hline WFO & Work for Others & 2.571 \\
\hline & & 0.144 \\
\hline
\end{tabular}

The following table lists the external organizations with the largest number of active HPC users, along with the number of INL users, as of April 22, 2020.

\begin{tabular}{|l|c|}
\hline \multicolumn{1}{|c|}{ Institution } & Active Users \\
\hline INL & 285 \\
\hline Idaho State University & 31 \\
\hline Oak Ridge National Laboratory & 21 \\
\hline University of Idaho & 20 \\
\hline Boise State University & 17 \\
\hline Argonne National Laboratory & 15 \\
\hline
\end{tabular}




\begin{tabular}{|l|c|}
\hline \multicolumn{1}{|c|}{ Institution } & Active Users \\
\hline Westinghouse Electric Company & 15 \\
\hline MPR Associates & 13 \\
\hline North Carolina State University & 11 \\
\hline Texas A\&M University & 11 \\
\hline Naval Nuclear Laboratory & 8 \\
\hline Nuclear Regulatory Commission & 8 \\
\hline University of Utah & 8 \\
\hline Massachusetts Institute of Technology & 7 \\
\hline Oregon State University & 7 \\
\hline University of Illinois at Urbana-Champaign & 7 \\
\hline
\end{tabular}

The following table displays the system utilization in million core-hours for the top 30 organizations using INL HPC systems from October 1, 2019, through March 31, 2020:

\begin{tabular}{|l|r|}
\hline \multicolumn{1}{|c|}{ Institution } & $\begin{array}{r}\text { Million } \\
\text { Core-Hours }\end{array}$ \\
\hline INL & 100.894 \\
\hline Boise State University & 12.988 \\
\hline Oak Ridge National Laboratory & 9.073 \\
\hline University of Idaho & 6.931 \\
\hline University of Michigan & 6.929 \\
\hline Westinghouse Electric Company & 6.549 \\
\hline North Carolina State University & 5.469 \\
\hline Los Alamos National Laboratory & 2.594 \\
\hline Massachusetts Institute of Technology & 2.418 \\
\hline University of Wyoming & 2.396 \\
\hline Purdue University & 2.378 \\
\hline University of Illinois at Urbana-Champaign & 2.144 \\
\hline MPR Associates & 1.873 \\
\hline Electric Power Research Institute & 1.695 \\
\hline University of New Mexico & 1.552 \\
\hline Texas A\&M University & 1.250 \\
\hline Framatome & 1.050 \\
\hline Seoul National University & 1.006 \\
\hline Virginia Tech & 0.976 \\
\hline University of Texas at Arlington & 0.814 \\
\hline Pennsylvania State University & 0.724 \\
\hline Idaho State University & 0.688 \\
\hline Kairos Power & 0.573 \\
\hline University of Nevada, Reno & 0.567 \\
\hline University of Utah & 0.532 \\
\hline
\end{tabular}




\begin{tabular}{|l|r|}
\multicolumn{1}{|c|}{ Institution } & $\begin{array}{c}\text { Million } \\
\text { Core-Hours }\end{array}$ \\
\hline University of Massachusetts Lowell & 0.501 \\
\hline University of Colorado Boulder & 0.444 \\
\hline University of South Carolina & 0.362 \\
\hline Utah State University & 0.357 \\
\hline University of Tennessee & 0.310 \\
\hline
\end{tabular}

As a result of the HPC funding provided by NSUF, several journal articles and conference proceedings were published. The following is a list of such published work:

\section{Journals}

Jin, M., Cao, P., \& Short, M. (2020). Achieving exceptional radiation tolerance with crystalline-amorphous nanocrystalline structures. Acta Materialia 186 587-596. https://doi.org/10.1016/j.actamat.2019.12.058

Jin, M., Cao, P., \& Short, M. (2019). Predicting the onset of void swelling in irradiated metals with machine learning. Journal of Nuclear Materials 523 189-197. https://doi.org/10.1016/j.jnucmat.2019.05.054

Liu, Y., Kochunas, B., Martin, W., \& Downar, T. (2019). Delayed Fission Energy Effect on LWR Normal Operation and Transients. Annals of Nuclear Energy 128 84-93. 10.1016/j.anucene.2018.12.048

Radaideh, M. (2019). Integrated framework for model assessment and advanced uncertainty quantification of nuclear computer codes under Bayesian statistics. Reliability Engineering \& System Safety 189 357-377. https://doi.org/10.1016/j.ress.2019.04.020

Ramirez Ruiz, J. (2019). The influence of plasticity-induced crack closure on creepfatigue crack growth in two heat-resistance steels. International Journal of Fatigue 125 291-298. https://doi.org/10.1016/j.ijfatigue.2019.04.007

Ramirez Ruiz, J. (2019). Fatigue and creep-fatigue crack growth in alloy 709 at elevated temperatures. Materials at High Temperatures 36 562-574.

https://doi.org/10.1080/09603409.2019.1664079

\section{Conferences}

Calvin, O., Ortensi, J., Schunert, S., Wang, Y., DeHart, M., \& Goluoglu, S. (August 2019). Implementation of Depletion Architecture in the MAMMOTH Reactor Physics Application, ANS Mathematics and Computational Methods 2019, Portland, OR, 201908-25.

Chen, J., \& Brooks, C. (March 2020). Experiment study of fluid motion and mass 
transfer in a cylindrical bubble column, ATH'2020 International Topical Meeting on Advances in Thermal Hydraulics, 2020-03-31.

Chen, J., \& Brooks, C. (August 2019). CFD simulation of Xenon removal by Helium bubble sparging in molten salt - effect of material properties, 18th International Topical Meeting on Nuclear Reactor Thermal Hydraulics (NURETH-18), 2019-08-18.

Hallee, B. (November 2019). Non-parametric Statistical Safety Analysis Tools to Support ATR Conversion to LEU, Reduced Enrichment for Research and Test Reactors, Zagreb, Croatia, 2019-10-06. Chicago, IL: https://www.rertr.anl.gov/RERTR40/pdfs/S13-P1_Hallee_paper.pdf

Khuwaileh, B., \& Turinsky, P. (August 2019). Bayesian Calibration of Cross-sections Using Plant Operating Data, M\&C 2019 Topical Meeting, Portland, OR, 2019-08-26.

Lee, Y., Gryazin, Y., \& Gonzales, R. (May 2019). Scalable high-resolution algorithms for landmine imaging problem, SPIE Defense + Commercial Sensing 2019 Exhibition, Baltimore, 2019-04-16. https://doi.org/10.117/12.2519782

Liu, W. (September 2019). Top Fuel 2019, BISON APPLICATION TO THE ANALYSIS OF LWR FUEL RESPONSES UNDER ACCIDENT CONDITIONS, Seattle, USA, 201909-22.

Liu, W. (September 2019). Top Fuel 2019, BISON AS A PCI SCREENING TOOL - PCI FAILURE MODEL DEVELOPMENT, Seattle, USA, 2019-09-22.

Liu, Y. (August 2019). Development of Simplified Methods for Ex-core Detector Response in MPACT, The International Conference on Mathematics and Computational Methods applied to Nuclear Science and Engineering (M\&C 2019), 2019-08-25. Portland, OR.

Liu, Y., \& et. al., (March 2020). An improved energy deposition model in MPACT and explicit heat generation coupling with CTF, Physor 2020, United Kingdom, 2020-03-29.

Liu, Y. (August 2019). Development of Simplified Methods for Ex-core Detector Response in MPACT, M\&C 2019, Portland, OR , 2019-08-25.

Mohamed, W., Ozaltun, H., \& Roh, H. (July 2019). Proceeding of the ASME 2019 Power Conference, ASME 2019 Power Conference, 2019-07-14.

Mohamed, W., Ozaltun, H., \& Roh, H. (July 2019). ASME 2019 Power conference, Effect of $\mathrm{Zr}$ diffusion barrier properties on the irradiation performance of $\mathrm{u}-10 \mathrm{mo}$ monolithic fuel plate, Snowbird, 2019-07-14. UT:

Ozaltun, H., Roh, H., \& Mohamed, W. (July 2019). Proceedings of the ASME 2019 Power Conference, ASME 2019 Power Conference, 2019-07-14.

Paladino, T., Nicholson, B., \& Nawotniak, S. (December 2019). AGU Conference 
Proceedings, Quantifying Wind Driven Collapse Scenarios of Explosive Eruption Plumes, 2019-12-09.

Patel, D. (March 2019). Studying the Calcium-dependent Albumin Conformation to Improve Osteoblast Cell Adhesion in Bone Repair and Regeneration, Biophysical Society (BPS) Conference, 2019-03-03.

Patel, D. (November 2019). Paired Simulations and Experimental Investigations into the Calcium-Dependent Bioactivity of Albumin, AIChE, 2019-11-14.

Rezwan, A., Jokisaari, A., \& Tonks, M. (April 2019). Modeling Fracture due to Thermal Expansion of Polycrystalline Alpha Uranium at Room Temperature, MRS Spring Meeting and Exhibits, Phoenix, 2019-04-22

Roh, H., Mohamed, W., \& Ozaltun, H. (July 2019). ASME 2019 Power conference, Effects of fission profiles on the performance of a U-10Mo fuel plate, Snowbird, 201907-14. UT:

Rykhlevskii, A., O'Grady, D., Kozlowski, T., \& Huff, K. (November 2019). Transactions of American Nuclear Society, The Impact of Xenon-135 on Load Following Transatomic Power Molten Salt Reactor, Washington D.C., 2019-11-17.

Wang, X., Liu, Y., Martin, W., \& Stimpson, S. (August 2019). Implementation of gamma transport capability in MPACT, M\&C 2019, Portland, OR, 2019-08-25. 